\title{
EXISTENCE OF GLOBAL BOUNDED WEAK SOLUTIONS TO A KEYFITZ-KRANZER SYSTEM*
}

\author{
YUN-GUANG $\mathrm{LU}^{\dagger}$ AND FENG GU
}

\begin{abstract}
In this paper, we study the global $L^{\infty}$ solutions for the Cauchy problem of the nonsymmetric Keyfitz-Kranzer system (1.1). In [Y.G. Lu, J. Funct. Anal., 261, 2797-2815, 2011], the same problem is studied when the nonlinear function $\phi(\rho, w)$ is of the form $\Phi(w)-P(\rho)$. In this paper, first we study the case $\phi(\rho, w)=f\left(\rho^{\alpha} \Phi(w)\right)$, where $\alpha>1$ is a constant and $\Phi(w)>0, f(r)$ are two suitable smooth functions of $w$ and the variable $r=\rho^{\alpha} \Phi(w)$. Using the compensated compactness method with the help of an $L^{1}$ estimate for $w^{\varepsilon}(\cdot, t)_{x}$, we first prove the pointwise convergence of the approximation functions $r^{\varepsilon}=\left(\rho^{\varepsilon}\right)^{\alpha} \Phi\left(w^{\varepsilon}\right)$. Then using a technique different from [Y.G. Lu, J. Funct. Anal., 261, 2797-2815, 2011], we prove the pointwise convergence of $\rho^{\varepsilon}$ and $w^{\varepsilon}$ respectively. Second, a similar existence result for systems of more than two equations is obtained when $\phi\left(\rho, w_{1}, w_{2}, \ldots w_{n}\right)=f\left(\rho^{\alpha} \Phi\left(w_{1}, w_{2}, \ldots w_{n}\right)\right)$. Third, we obtain a convergence framework of viscosity solutions of system (1.8) in Euler coordinates for a general nonlinear function $\phi\left(\rho, w_{1}, w_{2}, \ldots w_{n}\right)$ under the a priori estimate $0<c \leq \rho^{\varepsilon} \leq \frac{1}{c}, c>0$, motivated by the papers [L. Ambrosio, F. Bouchut, and C. De Lellis, Commun. PDE, 29(9-10), 1635-1651, 2004; L. Ambrosio, G. Crippa, A.Figalli, and L.A. Spinolo, SIAM J. Math. Anal., 41, 1890-1920, 2009; L. Ambrosio and C. De Lellis, Int. Math. Res. Not., 41, 2205-2220, 2003; A. Bressan, Rend. Sem. Mat. Univ. Padova, 110, 103-117, 2003] on multi-dimensional, symmetric systems of Keyfitz-Kranzer type and [E. Yu. Panov, Arch. Rat. Mech. Anal., 195, 643-673, 2010; E. Yu. Panov, J. Math. Sci. (N. Y.), 41, 159(2), 180-228, 2009] for multidimensional scalar conservation laws with discontinuous flux-functions.
\end{abstract}

Key words. Keyfitz-Kranzer system, compensated compactness theory, global solution.

AMS subject classifications. 35L65, $76 \mathrm{~N} 10$.

\section{Introduction}

In this paper, we study the Cauchy problem for the nonsymmetric system of Keyfitz-Kranzer type

$$
\left\{\begin{array}{l}
\rho_{t}+(\rho \phi(\rho, w))_{x}=0 \\
(\rho w)_{t}+(\rho w \phi(\rho, w))_{x}=0
\end{array}\right.
$$

with bounded measurable initial data

$$
(\rho(x, 0), w(x, 0))=\left(\rho_{0}(x), w_{0}(x)\right), \quad \rho_{0}(x) \geq 0,
$$

where

$$
\phi(\rho, w)=f(r), \quad r=\rho^{\alpha} \Phi(w),
$$

$\alpha>1$ is a constant, $\Phi(w)>0$ and $f(r)$ are suitable smooth functions of $w$, and the variable $r=\rho^{\alpha} \Phi(w)$. The more general form of (1.1) was first derived as a model for the elastic string by Keyfitz and Kranzer [12]. The global existence of weak solutions was well investigated in [11] for the chromatography system, and in $[7,8,12,13,14$, $17,20]$ for symmetric system

$$
\left(w_{i}\right)_{t}+\left(w_{i} \phi\left(w_{1}, w_{2}, \ldots w_{n}\right)\right)_{x}=0, \quad i=1,2, \ldots n,
$$

${ }^{*}$ Received: October 7, 2011; accepted (in revised form): February 6, 2012. Communicated by Alberto Bressan.

${ }^{\dagger}$ Department of Mathematics, Hangzhou Normal University, Hangzhou, P.R. CHINA, Tel. \& Fax: 0086-571-28865286, (yglu_2000@yahoo.com).

${ }^{\ddagger}$ Corresponding author, Department of Mathematics, Hangzhou Normal University, Hangzhou, P.R. CHINA, Tel. \& Fax: 0086-571-28865286, (gufengmath@yahoo.com.cn). 
where

$$
\phi(w)=\sum_{i=1}^{n} w_{i}^{l}, \quad l \geq 1
$$

or $\phi$ is a function only of the norm $\|w\|$. In connection with the symmetric KeyfitzKranzer system, the existence of solutions for multi-dimensional systems are studied in $[1,2,3,5]$ and references therein.

For the nonsymmetric system (1.1), as far as we know, the existence result of the Cauchy problem was studied in $[4,9,10,15]$ when $\phi(\rho, w)$ is of the form $\Phi(w)-P(\rho)$ for two suitable functions $\Phi(w)$ and $P(\rho)$. For a general function $\phi(\rho, w)$ with physically motivated conditions on the shape, the Cauchy problem of system (1.1) was studied when $\rho_{0}(x)$ and $w_{0}(x)$ are of bounded variation (cf. [23] for the details).

In this paper, we first study the case of $\phi(\rho, w)=f\left(\rho^{\alpha} \Phi(w)\right)$ and obtain the following theorem about the existence of global entropy solutions:

THEOREM 1.1. Let $\phi(\rho, w)=f\left(\rho^{\alpha} \Phi(w)\right)$, where $\alpha>1$ be a constant, the nonlinear function $f(r)$ is strictly increasing or decreasing on $r>0$, and the nonlinear function $\Phi(w)>c_{0}>0$ satisfies

$$
\alpha \Phi(w) \Phi^{\prime \prime}(w)-(\alpha-1)\left(\Phi^{\prime}(w)\right)^{2} \geq 0 .
$$

Let the initial data $\left(\rho_{0}(x), w_{0}(x)\right)$ be bounded, $\rho_{0}(x) \geq 0, w_{0 x}(x)$ be bounded in $L^{1}(R)$. Then the Cauchy problem (1.1)-(1.2) has a global bounded entropy solution $(\rho(x, t), w(x, t))$, where $w_{x}(\cdot, t)$ is bounded in $B V(R)$ in the sense of Lax, namely $(\rho(x, t), w(x, t))$ satisfies (1.1) in the sense of distributions and

$$
\int_{0}^{\infty} \int_{-\infty}^{\infty} \eta(\rho(x, t), w(x, t)) \theta_{t}+q(\rho(x, t), w(x, t)) \theta_{x} d x d t \geq 0,
$$

where $(\eta, q) \in C^{2}$ is a pair of entropy-entropy flux of system (1.1), $\eta$ is convex, and $\theta \in C_{0}^{\infty}\left(R \times R^{+}-\{t=0\}\right)$ is a nonnegative function.

Note 1. The conditions of $\Phi(w)$ in (1.6) are satisfied for the function $\Phi(w)=w^{\beta}$ when $w \geq c_{0}>0, \beta \geq \alpha$.

Second, a similar existence result for systems of more than two equations is obtained when $\phi\left(\rho, w_{1}, w_{2}, \ldots w_{n}\right)=f\left(\rho^{\alpha} \Phi\left(w_{1}, w_{2}, \ldots w_{n}\right)\right)$.

Consider

$$
\left\{\begin{array}{l}
\rho_{t}+\left(\rho \phi\left(\rho, w_{1}, w_{2}, \ldots w_{n}\right)\right)_{x}=0, \\
\left(\rho w_{i}\right)_{t}+\left(\rho w_{i} \phi\left(\rho, w_{1}, w_{2}, \ldots w_{n}\right)\right)_{x}=0, \quad i=1,2, \ldots n
\end{array}\right.
$$

with bounded measurable initial data

$$
\left(\rho(x, 0), w_{i}(x, 0)\right)=\left(\rho_{0}(x), w_{i 0}(x)\right), \quad \rho_{0}(x) \geq 0, \quad i=1,2, \ldots n .
$$

ThEOREM 1.2. Let $\phi\left(\rho, w_{1}, w_{2}, \ldots w_{n}\right)=f\left(\rho^{\alpha} \Phi\left(w_{1}, w_{2}, \ldots w_{n}\right)\right)$, where $\alpha>1$ is a constant, the nonlinear function $f(r)$ is strictly increasing or decreasing on $r>0$, and the nonlinear function $\Phi\left(w_{1}, w_{2}, \ldots w_{n}\right)=\Phi(w)>c_{0}>0$ satisfies

$$
\alpha(\alpha-1) \rho^{\alpha-2} \Phi(w) \rho_{x}^{2}+2(\alpha-1) \rho^{\alpha-1} \sum_{i=1}^{n} \Phi(w)_{w_{i}} w_{i x} \rho_{x}+\sum_{i, j=1}^{n} \rho^{\alpha} \Phi(w)_{w_{i} w_{j}} w_{i x} w_{j x} \geq 0 .
$$


Let the initial data $\left(\rho_{0}(x), w_{i 0}(x)\right)$ be bounded, $\rho_{0}(x) \geq 0$, and $\frac{d w_{i 0}}{d x}$ be bounded in $L^{1}(R)$. Then the Cauchy problem (1.8)-(1.9) has a global bounded entropy solution $\left(\rho(x, t), w_{i}(x, t)\right)$, where the $w_{i x}(\cdot, t)$ are bounded in $B V(R)$.

Note 2. In general, when $\alpha>1$, the conditions in (1.10) are satisfied if the function $\rho^{\alpha} \Phi(w)$ is convex about the variables $(\rho, w)$. In fact, if $\rho^{\alpha} \Phi(w)$ is convex, the following inequality is true:

$$
\alpha(\alpha-1) \rho^{\alpha-2} \Phi(w) \rho_{x}^{2}+2 \alpha \rho^{\alpha-1} \sum_{i=1}^{n} \Phi(w)_{w_{i}} w_{i x} \rho_{x}+\sum_{i, j=1}^{n} \rho^{\alpha} \Phi(w)_{w_{i} w_{j}} w_{i x} w_{j x} \geq 0,
$$

and the coefficient of the term $\rho^{\alpha-1} \sum_{i=1}^{n} \Phi(w)_{w_{i}} w_{i x} \rho_{x}$ in (1.10) is $\alpha-1$ and could be more easily controlled by other terms.

It is worthwhile to remark that if the solution has the bound $\rho>0$ away from vacuum, a more enlightening way to study system (1.1), for a general $\phi(\rho, w)$ strictly monotone in $\rho$, is to introduce a Lagrangian transformation [20]

$$
t=s, \quad \frac{\partial y}{\partial x}=\rho, \quad \frac{\partial y}{\partial t}=-\rho \phi
$$

and reduce the system to

$$
\left\{\begin{array}{l}
w_{s}=0 \\
\left(\frac{1}{\rho}\right)_{s}-\phi(\rho, w)_{y}=0
\end{array}\right.
$$

System (1.13) is equivalent to a scalar equation with a discontinuous flux-function, so the results of Panov [18, 19] about the existence of entropy solutions for multidimensional scalar conservation laws with discontinuous flux-functions can be applied fairly generally. To prove the existence of global solutions of system (1.13), Panov assumes a technical condition $\phi(a, w)=\phi(b, w)=0$ for some $0<a<b$ for obtaining the a priori estimate $0<a \leq \rho^{\varepsilon} \leq b$. From our research in [15], when system (1.1) is in Euler coordinates, the bound of the viscosity solutions $\rho^{\varepsilon}$ can be obtained for more general function $\phi$ by the invariant region theorem.

Based on the above analysis, although (1.1) and (1.13) are equivalent when $\rho>0$ and the global solutions of system (1.13) were well studied by Panov in [18, 19], we are still interested in studying the convergence of viscosity solutions of (1.1) and give a different, short proof of the following theorem.

TheOrem 1.3. Let the initial data $\left(\rho_{0}(x), w_{i 0}(x)\right)$ satisfy

$$
0<\delta \leq \rho_{0}(x) \leq M_{1}, \quad-M_{2} \leq w_{i 0}(x) \leq M_{3}
$$

for some constants $\delta, M_{j}, j=1,2,3$, assume that the total variation of $w_{i 0}(x)$ be bounded, and for any fixed constants $c_{j}, j=1,2, \ldots n$, and $-M_{2} \leq c_{j} \leq M_{3}$, assume that the function $f(\rho)=\phi\left(\rho, c_{1}, c_{2}, \ldots c_{n}\right)$ satisfies

$$
f \in C^{2}\left(R^{+}\right), \quad \text { meas }\left\{r: 2 f^{\prime}(r)+r f^{\prime \prime}(r)=0\right\}=0 .
$$

If the viscosity solutions $\rho^{\varepsilon}$ of the Cauchy problem (2.26) and (1.9) have the a-priori $L^{\infty}$ estimate

$$
0<c_{1} \leq \rho^{\varepsilon} \leq c_{2}
$$


for suitable positive constants $c_{1}$ and $c_{2}$ independent of $\varepsilon$, then there exists a subsequence of solutions $\left(\rho^{\varepsilon}, w_{i}^{\varepsilon}\right)$ (still denoted $\left(\rho^{\varepsilon}, w_{i}^{\varepsilon}\right)$ ), of the Cauchy problem (2.26) and (1.9), which converges pointwise to functions $\left(\rho, w_{i}\right)$ and the limit $\left(\rho, w_{i}\right)$ is a weak entropy solution of the Cauchy problem (1.8)-(1.9) in the sense of Lax, where the $w_{i x}(\cdot, t)$ are bounded in $B V(R)$.

Note 3. Theorem 1.3 is an extension of Panov's result in $[18,19]$. We have the existence of the global solution of system (1.1) so long as we have the estimate (1.16) for some conditions on $\phi$. For instance, as we did in Section 3 in [15], we add a factor $\rho-\delta$ before the function $w-P(\rho)$ and study the case of $\phi(\rho, w)=(\rho-\delta)(w-P(\rho))$, then repeating the proof in [15] we have the positive, lower estimate $\rho^{\varepsilon} \geq \delta>0$ and the bound from above $\rho^{\varepsilon} \leq M$ by using the invariant region theorem, for any fixed $\delta$.

We will prove Theorems 1.1-1.3 in the next section. The main technique is the compensated compactness method coupled with the artificial viscosity.

\section{Proofs of Theorems 1.1-1.3}

Consider the Cauchy problem for the related parabolic system

$$
\left\{\begin{array}{l}
\rho_{t}+(\rho \phi(\rho, w))_{x}=\varepsilon \rho_{x x} \\
(\rho w)_{t}+(\rho w \phi(\rho, w))_{x}=\varepsilon(\rho w)_{x x}
\end{array}\right.
$$

with initial data

$$
\left(\rho^{\varepsilon}(x, 0), w^{\varepsilon}(x, 0)\right)=\left(\rho_{0}(x)+\varepsilon, w_{0}(x)\right), \quad \rho_{0}(x) \geq 0,
$$

where $\left(\rho_{0}(x), w_{0}(x)\right)$ is given by $(1.2)$.

First, since $\rho^{\varepsilon}(x, 0) \geq \varepsilon>0$, we may choose a small time $t_{1}$ such that the local solution $\rho^{\varepsilon}(x, t)$, of the Cauchy problem (2.1), and (2.2), has the lower bound $\rho^{\varepsilon}(x, t) \geq$ $\frac{\varepsilon}{2}$ for $t \in\left[0, t_{1}\right]$. After we have the a priori estimates given in (2.4), (2.7) and (2.8), we may extend the local solution step by step to obtain a global solution. This process is standard.

Substituting the first equation in (2.1) into the second, we have

$$
w_{t}+\phi(\rho, w) w_{x}=\varepsilon w_{x x}+\frac{2 \varepsilon}{\rho} \rho_{x} w_{x} .
$$

By applying the maximum principle to (2.3), we first have the estimate

$$
-M_{2} \leq w^{\varepsilon} \leq M_{3}
$$

for two suitable constants $M_{2}, M_{3}$, which depend only on the initial date, but are independent of $\varepsilon$.

We multiply the first equation in (2.1) by $r_{\rho}$ and the equation (2.3) by $r_{w}$, then add the results to obtain

$$
\begin{aligned}
& r_{t}+\phi(r) r_{x}+\rho r_{\rho} \phi^{\prime}(r) r_{x} \\
= & \varepsilon r_{x x}-\varepsilon\left(r_{\rho \rho} \rho_{x}^{2}+2 r_{\rho w} \rho_{x} w_{x}+r_{w w} w_{x}^{2}\right)+\frac{2 \varepsilon}{\rho} \rho_{x} r_{w} w_{x} \\
= & \varepsilon r_{x x}-\varepsilon\left(\alpha(\alpha-1) \rho^{\alpha-2} \Phi(w) \rho_{x}^{2}+2(\alpha-1) \rho^{\alpha-1} \Phi^{\prime}(w) \rho_{x} w_{x}+\rho^{\alpha} \Phi^{\prime \prime}(w) w_{x}^{2}\right) \\
\leq & \varepsilon r_{x x}
\end{aligned}
$$

due to the condition (1.6). 
By applying the maximum principle to (2.5), we have the estimate

$$
r^{\varepsilon} \leq M
$$

and hence the estimate

$$
\rho^{\varepsilon} \leq M_{1}
$$

due to the conditions in Theorem 1.1.

Since $\phi(\rho, w)=f\left(\rho^{\alpha} \Phi(w)\right), \alpha>1$, and $\Phi(w)>0, \phi(\rho, w)$ is bounded. Therefore, using an argument by Bereux and Sainsaulieu (cf. [15]), we have the following positive, lower bound estimate on $\rho^{\varepsilon}$ :

$$
\rho^{\varepsilon} \geq c(t, \varepsilon)>0, \quad \text { since } \quad \rho_{0}^{\varepsilon}(x) \geq \varepsilon>0,
$$

where $c(t, \varepsilon)$ could tend to zero as the time $t$ tends to infinity or $\varepsilon$ tends to zero.

The estimates in (2.4), (2.7), and (2.8) ensure the existence of viscosity solutions $\left(\rho^{\varepsilon}, w^{\varepsilon}\right)$ of the Cauchy problem (2.1) and (2.2) for any fixed $\varepsilon>0$.

Now we prove the pointwise convergence of $\left(\rho^{\varepsilon}, w^{\varepsilon}\right)$ as $\varepsilon$ goes to zero.

We rewrite (2.5) as

$$
r_{t}+\phi(r) r_{x}+\alpha r \phi^{\prime}(r) r_{x}=\varepsilon r_{x x}-I
$$

due to $\rho r_{\rho}=\alpha r$, where

$$
I=\varepsilon\left(\alpha(\alpha-1) \rho^{\alpha-2} \Phi(w) \rho_{x}^{2}+2(\alpha-1) \rho^{\alpha-1} \Phi^{\prime}(w) \rho_{x} w_{x}+\rho^{\alpha} \Phi^{\prime \prime}(w) w_{x}^{2}\right)
$$

is nonnegative due to the condition (1.6), and so we can prove that $I$ is bounded in $L_{l o c}^{1}\left(R \times R^{+}\right)$if we multiply $(2.9)$ by a suitable test function.

The left-hand side of (2.9) has only the variable $r$, so we may use Tartar's result [22] on the scalar equation to prove the pointwise convergence of $r^{\varepsilon}$ :

$$
r^{\varepsilon}(x, t) \rightarrow l(x, t), \quad \text { as } \quad \varepsilon \rightarrow 0,
$$

for a bounded function $l(x, t)$.

To prove the pointwise convergence of $\rho^{\varepsilon}$, we rewrite the first equation in system $(2.1)$ as

$$
\rho_{t}+\rho_{x}\left(f(r)+\alpha \rho^{\alpha} \Phi(w) f^{\prime}(r)\right)+\rho^{\alpha+1} \Phi^{\prime}(w) f^{\prime}(r) w_{x}=\varepsilon \rho_{x x} .
$$

Using the equation (2.3) and the same technique given in [15] or [21], we have the estimate

$$
\int_{-\infty}^{\infty}\left|w_{x}\right|(x, t) d x \leq \int_{-\infty}^{\infty}\left|w_{x}\right|(x, 0) d x \leq M .
$$

Let $\rho^{\alpha}=u$. We multiply (2.12) by $\alpha \rho^{\alpha-1}$ to obtain

$$
u_{t}+u_{x}\left[f(u \Phi)+\alpha u \Phi f^{\prime}(u \Phi)\right]=\varepsilon u_{x x}-\alpha(\alpha-1) \rho^{\alpha-2} \rho_{x}^{2}-\alpha u^{2} f^{\prime}(u \Phi) \Phi_{x},
$$

or

$$
\begin{aligned}
& u_{t}+\left(\int_{0}^{u}\left[f(\tau \Phi)+\alpha \tau \Phi f^{\prime}(\tau \Phi)\right] d \tau\right)_{x} \\
= & \varepsilon u_{x x}-\varepsilon \alpha(\alpha-1) \rho^{\alpha-2} \rho_{x}^{2}-\alpha u^{2} f^{\prime}(u \Phi) \Phi_{x}+\left(\int_{0}^{u}\left[f(\tau \Phi)+\alpha \tau \Phi f^{\prime}(\tau \Phi)\right] d \tau\right)_{\Phi} \Phi_{x} .
\end{aligned}
$$


Since the estimate in (2.13), the terms

$$
-\alpha u^{2} f^{\prime}(u \Phi) \Phi_{x}+\left(\int_{0}^{u}\left[f(\tau \Phi)+\alpha \tau \Phi f^{\prime}(\tau \Phi)\right] d \tau\right)_{\Phi} \Phi_{x}
$$

from the right-hand side of (2.15) are bounded in $L_{l o c}^{1}\left(R \times R^{+}\right)$. Multiplying (2.15) by a suitable test function, we have that the

$$
\varepsilon \rho^{\alpha-2} \rho_{x}^{2} \quad \text { are bounded in } \quad L_{l o c}^{1}\left(R \times R^{+}\right) .
$$

So we can use Murat's theorem [16] to prove that the left-hand side of (2.15)

$$
u_{t}^{\varepsilon}+\left(\int_{0}^{u^{\varepsilon}}\left[f(\tau \Phi)+\alpha \tau \Phi f^{\prime}(\tau \Phi)\right] d \tau\right)_{x} \text { are compact in } H_{l o c}^{-1}\left(R \times R^{+}\right) .
$$

By simple calculation,

$$
\begin{aligned}
& \int_{0}^{u}\left[f(\tau \Phi)+\alpha \tau \Phi f^{\prime}(\tau \Phi)\right] d \tau=\frac{1}{\Phi} \int_{0}^{u}\left[f(\tau \Phi)+\alpha \tau \Phi f^{\prime}(\tau \Phi)\right] d(\tau \Phi) \\
= & \frac{1}{\Phi} \int_{0}^{u \Phi}\left[f(\tau)+\alpha \tau f^{\prime}(\tau)\right] d \tau=\frac{\rho^{\alpha}}{r} \int_{0}^{r}\left[f(\tau)+\alpha \tau f^{\prime}(\tau)\right] d \tau .
\end{aligned}
$$

Similarly, we can prove that the left-hand side of the first equation in (2.1)

$$
\rho_{t}^{\varepsilon}+\left(\rho^{\varepsilon} f\left(r^{\varepsilon}\right)\right)_{x} \quad \text { are compact in } \quad H_{l o c}^{-1}\left(R \times R^{+}\right) .
$$

So, applying the Curl-Div theorem to the pairs of functions

$$
\left(\rho^{\varepsilon}, \rho^{\varepsilon} f\left(r^{\varepsilon}\right)\right)
$$

and

$$
\left(\left(\rho^{\varepsilon}\right)^{\alpha}, \frac{\left(\rho^{\varepsilon}\right)^{\alpha}}{r^{\varepsilon}} \int_{0}^{r^{\varepsilon}}\left[f(\tau)+\alpha \tau f^{\prime}(\tau)\right] d \tau\right)
$$

we have

$$
\begin{aligned}
& \left(\frac{1}{l(x, t)} \int_{0}^{l(x, t)}\left[f(\tau)+\alpha \tau f^{\prime}(\tau)\right] d \tau-f(l(x, t))\right) \overline{\left(\rho^{\varepsilon}\right)^{\alpha+1}} \\
= & \left(\frac{1}{l(x, t)} \int_{0}^{l(x, t)}\left[f(\tau)+\alpha \tau f^{\prime}(\tau)\right] d \tau-f(l(x, t))\right) \overline{\rho^{\varepsilon}} \overline{\left(\rho^{\varepsilon}\right)^{\alpha}}
\end{aligned}
$$

due to the pointwise convergence of $r^{\varepsilon}(x, t) \rightarrow l(x, t)$ and $\rho^{\varepsilon} \geq 0$, where $\overline{\theta^{\varepsilon}}$ denotes the weak-star limit of $\theta^{\varepsilon}$.

Since $f(r)$ is strictly increasing or decreasing on $r>0$,

$$
\frac{1}{r} \int_{0}^{r}\left[f(\tau)+\alpha \tau f^{\prime}(\tau)\right] d \tau-f(r)=0 \quad \text { only on } \quad r=0 .
$$

Therefore we have from $(2.20)$

$$
\overline{\left(\rho^{\varepsilon}\right)^{\alpha+1}}=\overline{\rho^{\varepsilon}} \overline{\left(\rho^{\varepsilon}\right)^{\alpha}}
$$


and so $\rho^{\varepsilon} \rightarrow \rho(x, t)$ a.e. on the region $r>0$.

To prove the pointwise convergence of $w^{\varepsilon}$, we first have that both $w_{x}^{\varepsilon}$ and $\left(\left(w^{\varepsilon}\right)^{2}\right)_{x}$ are compact in $H_{l o c}^{-1}\left(R \times R^{+}\right)$since they are bounded both in $L_{l o c}^{1}\left(R \times R^{+}\right)$and in $W^{-1, \infty}\left(R \times R^{+}\right)$. Thus we may apply the Curl-Div Theorem to the pairs of functions

$$
\left(\rho^{\varepsilon}, \rho^{\varepsilon} f\left(r^{\varepsilon}\right)+w^{\varepsilon}\right)
$$

and

$$
\left(\rho^{\varepsilon} w^{\varepsilon}, \rho^{\varepsilon} w^{\varepsilon} f\left(r^{\varepsilon}\right)+\left(w^{\varepsilon}\right)^{2}\right)
$$

to obtain

$$
\overline{\rho^{\varepsilon}}\left(\overline{\rho^{\varepsilon} w^{\varepsilon} f\left(r^{\varepsilon}\right)+\left(w^{\varepsilon}\right)^{2}}\right)-\left(\overline{\rho^{\varepsilon} w^{\varepsilon}}\right)\left(\overline{\rho^{\varepsilon} f\left(r^{\varepsilon}\right)+w^{\varepsilon}}\right)=0 .
$$

By the pointwise convergence of $\rho^{\varepsilon}$ and $f\left(r^{\varepsilon}\right)$, we have

$$
\rho\left(\overline{\left(w^{\varepsilon}\right)^{2}}-\left(\overline{w^{\varepsilon}}\right)^{2}\right)=0,
$$

which includes the pointwise convergence of $w^{\varepsilon}$ on the region $\rho>0$. Thus, we complete the proof of Theorem 1.1.

Proof of Theorem 1.2. The proof of Theorem 1.2 is same to that of Theorem 1.1 except the reduction of (2.5).

We add the viscosity terms to the right-hand side of system (1.8) and consider the related parabolic system

$$
\left\{\begin{array}{l}
\rho_{t}+\left(\rho \phi\left(\rho, w_{1}, w_{2}, \ldots w_{n}\right)\right)_{x}=\varepsilon \rho_{x x} \\
\left(\rho w_{i}\right)_{t}+\left(\rho w_{i} \phi\left(\rho, w_{1}, w_{2}, \ldots w_{n}\right)\right)_{x}=\varepsilon\left(\rho w_{i}\right)_{x x}, \quad i=1,2, \ldots n
\end{array}\right.
$$

Substituting the first equation in (2.26) into the second, we have

$$
w_{i t}+\phi(\rho, w) w_{i x}=\varepsilon w_{i x x}+\frac{2 \varepsilon}{\rho} \rho_{x} w_{i x} .
$$

We multiply the first equation in (2.26) by $r_{\rho}$ and equation (2.27) by $r_{w_{i}}$, then add the results to obtain

$$
\begin{aligned}
& r_{t}+\phi(r) r_{x}+\rho r_{\rho} \phi^{\prime}(r) r_{x} \\
= & \varepsilon r_{x x}-\varepsilon\left(r_{\rho \rho} \rho_{x}^{2}+2 \sum_{i=1}^{n} r_{\rho w_{i}} \rho_{x} w_{i x}+\sum_{i, j=1}^{n} r_{w_{i} w_{j}} w_{i x} w_{j x}\right)+\frac{2 \varepsilon}{\rho} \rho_{x} \sum_{i=1}^{n} r_{w_{i}} w_{i x} \\
= & \varepsilon r_{x x}-\varepsilon\left(\alpha(\alpha-1) \rho^{\alpha-2} \Phi \rho_{x}^{2}+2(\alpha-1) \rho^{\alpha-1} \sum_{i=1}^{n} \Phi_{w_{i}} w_{i x} \rho_{x}+\sum_{i, j=1}^{n} \rho^{\alpha} \Phi_{w_{i} w_{j}} w_{i x} w_{j x}\right) \\
\leq & \varepsilon r_{x x}
\end{aligned}
$$

due to the condition (1.10) in Theorem 1.2. So, we may obtain the uniform $L^{\infty}$ estimate of $\left(\rho^{\varepsilon}, w_{i}^{\varepsilon}\right)$, and so the existence of the viscosity solutions of the parabolic system (2.26). Similarly to the proof of Theorem 1.1, we may prove the pointwise convergence of $r^{\varepsilon}$ first by using the left-hand side of equation (2.28) coupled with Tartar's result on the scalar equation. Repeating the process of the proof of Theorem 1.1, we may prove the pointwise convergence of $\rho^{\varepsilon}$ and $w_{i}^{\varepsilon}$, and complete the proof of Theorem 1.2. 
Proof of Theorem 1.3. We prove Theorem 1.3 in the following several steps. First, using equation (2.27), we have the estimates

$$
-M_{2} \leq w_{i}^{\varepsilon}(x, t) \leq M_{3}, \quad \int_{-\infty}^{\infty}\left|w_{i x}\right|(x, t) d x \leq M
$$

due to the conditions in Theorem 1.3.

We multiply the first equation in $(2.26)$ by $g^{\prime}(\rho)$ to obtain

$$
g(\rho)_{t}+g^{\prime}(\rho)\left(\phi+\rho \phi_{\rho}\right) \rho_{x}+g^{\prime}(\rho) \rho \sum_{i=1}^{n} \phi_{w_{i}} w_{i x}=\varepsilon g(\rho)_{x x}-\varepsilon g^{\prime \prime}(\rho) \rho_{x}^{2}
$$

or

$$
\begin{aligned}
& g(\rho)_{t}+\left(\int_{0}^{\rho} g^{\prime}(s)\left(\phi\left(s, w_{1}, w_{2}, \ldots w_{n}\right)+s \phi_{\rho}\left(s, w_{1}, w_{2}, \ldots w_{n}\right)\right) d s\right)_{x} \\
= & \varepsilon g(\rho)_{x x}-\varepsilon g^{\prime \prime}(\rho) \rho_{x}^{2}-g^{\prime}(\rho) \rho \sum_{i=1}^{n} \phi_{w_{i}} w_{i x} \\
& +\sum_{i=1}^{n}\left(\int_{0}^{\rho} g^{\prime}(s)\left(\phi\left(s, w_{1}, w_{2}, \ldots w_{n}\right)+s \phi_{\rho}\left(s, w_{1}, w_{2}, \ldots w_{n}\right)\right) d s\right)_{w_{i}} w_{i x} .
\end{aligned}
$$

By the second estimate in (2.29), the last two terms on the right-hand side of (2.31) are bounded in $L_{l o c}^{1}\left(R \times R^{+}\right)$, so we may choose a strictly convex function $g(\rho)$ to obtain that

$$
\varepsilon\left(\rho_{x}^{\varepsilon}\right)^{2} \quad \text { are bounded in } \quad L_{l o c}^{1}\left(R \times R^{+}\right)
$$

with the help of (2.31).

Let $\eta\left(\rho, w_{1}, w_{2}, \ldots w_{n}\right)=\rho F\left(w_{1}, w_{2}, \ldots w_{n}\right)$, where $F$ is a strictly convex function. We multiply the first equation in $(2.26)$ by $\eta_{\rho},(2.27)$ by $\eta_{w_{i}}$, and add the results to obtain

$$
\begin{aligned}
& \eta\left(\rho, w_{1}, w_{2}, \ldots w_{n}\right)_{t}+\left(\phi \eta\left(\rho, w_{1}, w_{2}, \ldots w_{n}\right)\right)_{x}=\varepsilon \eta\left(\rho, w_{1}, w_{2}, \ldots w_{n}\right)_{x x} \\
& -\varepsilon \rho \sum_{i, j=1}^{n} F_{w_{i} w_{j}} w_{i x} w_{j x} \leq \varepsilon \eta\left(\rho, w_{1}, w_{2}, \ldots w_{n}\right)_{x x}-\varepsilon c_{0} \rho \sum_{i=1}^{n} w_{i x}^{2}
\end{aligned}
$$

for a suitable positive constant $c_{0}$. Thus we obtain that

$$
\varepsilon \rho \sum_{i=1}^{n} w_{i x}^{2} \quad \text { are bounded in } \quad L_{l o c}^{1}\left(R \times R^{+}\right) .
$$

If we have the a priori $L^{\infty}$ estimate (1.16), we have from (2.32) and (2.34) that the first term in the right-hand side of (2.27) is compact in $H_{l o c}^{-1}\left(R \times R^{+}\right)$, the second term is bounded in $L_{l o c}^{1}\left(R \times R^{+}\right)$, and from (2.29) that the second term in the lefthand side is bounded in $L_{l o c}^{1}\left(R \times R^{+}\right)$. So, we can prove that the first term in the left-hand side of (2.27)

$$
w_{i t}^{\varepsilon} \quad \text { are compact in } \quad H_{l o c}^{-1}\left(R \times R^{+}\right) .
$$


Therefore both $\left(w_{i}^{\varepsilon}\right)_{t}+\left(\left(w_{i}^{\varepsilon}\right)^{2}\right)_{x}$ and $\left(\left(w_{i}^{\varepsilon}\right)^{2}\right)_{t}+\frac{4}{3}\left(\left(w_{i}^{\varepsilon}\right)^{3}\right)_{x}$ are compact in $H_{l o c}^{-1}(R \times$ $\left.R^{+}\right)$. Using Tartar's result on the scalar equation again, we obtain the pointwise convergence of $w_{i}^{\varepsilon}$.

It remains to prove the pointwise convergence of $\rho^{\varepsilon}$.

We rewrite the first equation in (2.26) as

$$
\rho_{t}+\left(\phi(\rho, w)+\rho \phi_{\rho}(\rho, w)\right) \rho_{x}+\rho \sum_{i=1}^{n} \phi_{w_{i}}(\rho, w) w_{i x}=\varepsilon \rho_{x x}
$$

or

$$
\rho_{t}+F(\rho, w)_{x}=\varepsilon \rho_{x x}-\rho \sum_{i=1}^{n} \phi_{w_{i}}(\rho, w) w_{i x}-\sum_{i=1}^{n} F_{w_{i}}(\rho, w) w_{i x}
$$

where $F(\rho, w)=\int_{0}^{\rho} \phi(s, w)+s \phi_{\rho}(s, w) d s$. Since the last two terms in the right-hand side of $(2.37)$ are bounded in $L_{l o c}^{1}\left(R \times R^{+}\right)$, we can prove that

$$
\rho_{t}^{\varepsilon}+F\left(\rho^{\varepsilon}, w^{\varepsilon}\right)_{x} \quad \text { are compact in } \quad H_{l o c}^{-1}\left(R \times R^{+}\right) .
$$

Similarly, we multiply $(2.36)$ by $F_{\rho}(\rho, w),(2.27)$ by $F_{w_{i}}(\rho, w)$, and add the results to obtain

$$
\begin{aligned}
& F(\rho, w)_{t}+F_{\rho}^{2}(\rho, w) \rho_{x} \\
= & \varepsilon F(\rho, w)_{x x}-\varepsilon F_{\rho \rho}(\rho, w) \rho_{x}^{2} \\
& -2 \varepsilon \sum_{i=1}^{n} F_{\rho w_{i}}(\rho, w) \rho_{x} w_{i x}-\varepsilon \sum_{i, j=1}^{n} F_{w_{i} w_{j}}(\rho, w) w_{i x} w_{j x} \\
& -\rho F_{\rho}(\rho, w) \sum_{i=1}^{n} F_{w_{i}}(\rho, w) w_{i x}+\varepsilon\left(\frac{2 \rho_{x}}{\rho}-\phi(\rho, w)\right) \sum_{i=1}^{n} F_{w_{i}}(\rho, w) w_{i x}
\end{aligned}
$$

or

$$
\begin{aligned}
& F(\rho, w)_{t}+\left(\int_{0}^{\rho} F_{\rho}^{2}(s, w) d s\right)_{x} \\
=\varepsilon & F(\rho, w)_{x x}-\varepsilon F_{\rho \rho}(\rho, w) \rho_{x}^{2} \\
& -2 \varepsilon \sum_{i=1}^{n} F_{\rho w_{i}}(\rho, w) \rho_{x} w_{i x}-\varepsilon \sum_{i, j=1}^{n} F_{w_{i} w_{j}}(\rho, w) w_{i x} w_{j x} \\
& +\left(-\rho F_{\rho}(\rho, w)+\varepsilon\left(\frac{2 \rho_{x}}{\rho}-\phi(\rho, w)\right)\right) \sum_{i=1}^{n} F_{w_{i}}(\rho, w) w_{i x} \\
& +\sum_{i=1}^{n}\left(\int_{0}^{\rho} F_{\rho}^{2}(s, w) d s\right)_{w_{i}} w_{i x} .
\end{aligned}
$$

Thus, using the estimates given in (1.16), (2.29), (2.32), and (2.34), we can prove that

$$
F\left(\rho^{\varepsilon}, w^{\varepsilon}\right)_{t}+\left(\int_{0}^{\rho^{\varepsilon}} F_{\rho}^{2}\left(s, w^{\varepsilon}\right) d s\right)_{x} \quad \text { are compact in } \quad H_{l o c}^{-1}\left(R \times R^{+}\right) .
$$

Using the Curl-Div Theorem in the compensated compactness theory $([16,22])$ to the function pairs given in (2.38) and (2.41), and by paying attention to the pointwise 
convergence of $w_{i}^{\varepsilon}$ and the condition in (1.15), we obtain the pointwise convergence of $\rho^{\varepsilon}$ and so complete the proof of Theorem 1.3.

Acknowledgment. The authors thank the reviewers for many valuable comments and suggestions, which led to a substantial improvement of the paper. This paper is partially supported by NNSF No. 11071169 of China and a Humboldt fellowship.

\section{REFERENCES}

[1] L. Ambrosio, F. Bouchut, and C. De Lellis, Well-posedness for a class of hyperbolic systems of conservation laws in several space dimensions, Commun. PDE, 29(9-10), 1635-1651, 2004.

[2] L. Ambrosio, G. Crippa, A.Figalli, and L.A. Spinolo, Some new well-posedness results for continuity and transport equations, and applications to the chromatography system, SIAM J. Math. Anal., 41, 1890-1920, 2009.

[3] L. Ambrosio and C. De Lellis, Existence of solutions for a class of hyperbolic systems of conservation laws in several space dimensions, Int. Math. Res. Not., 41, 2205-2220, 2003.

[4] A. Aw and M. Rascle, Resurrection of "second order" models of traffic flow, SIAM J. Appl. Math., 60, 916-938, 2000.

[5] A. Bressan, An ill posed Cauchy problem for a hyperbolic system in two space dimensions, Rend. Sem. Mat. Univ. Padova, 110, 103-117, 2003.

[6] A. Bressan and W. Shen, BV estimate for multicomponent chromatography with relaxation, Disc. Cont. Dyn. Sys., 6, 21-38, 2000.

[7] G.Q. Chen, Hyperbolic system of conservation laws with a symmetry, Commun. PDE, 16, 1461-1487, 1991.

[8] H. Freistuhler, On the Cauchy problem for a class of hyperbolic systems of conservation laws, J. Diff. Eqs., 112, 170-178, 1994.

[9] M. Garavello and B. Piccoli, Traffic flow on a road network using the Aw-Rascle model, Commun. Part. Diff. Equ., 31, 243-275, 2006.

[10] M. Godvik and H. Hanche-Olsen, Existence of solutions for the AW-Rascle traffic flow model with vacuum, J. Hyp. Diff. Equ., 5, 45-64, 2008.

[11] F. James, Y.J. Peng, and B. Perthame, Kinetic formulation for chromatography and some other hyperbolic systems, J. Math. Pure Appl., 74, 367-385, 1995.

[12] B. Keyfitz and H. Kranzer, A system of nonstrictly hyperbolic conservation laws arising in elasticity, Arch. Rat. Mech. Anal., 72, 219-241, 1980.

[13] A. Kearsley and A. Reiff, Existence of weak solutions to a class of nonstrictly hyperbolic conservation laws with non-interacting waves, Pacific J. Math., 205, 153-170, 2002.

[14] T.P. Liu and J.H. Wang, On a hyperbolic system of conservation laws which is not strictly hyperbolic, J. Diff. Eqs., 57, 1-14, 1985.

[15] Y.G. Lu, Existence of global bounded weak solutions to a non-symmetric system of KeyfitzKranzer type, J. Funct. Anal., 261, 2797-2815, 2011.

[16] F. Murat, Compacité par compensation, Ann. Scuola Norm. Sup. Pisa, 5, 489-507, 1978.

[17] E. Yu. Panov, On the theory of generalized entropy solutions of the Cauchy problem for a class of non-strictly hyperbolic systems of conservation laws, Sbornik: Mathematics, 191, 121-150, 2000.

[18] E. Yu. Panov, Existence and strong pre-compactness properties for entropy solutions of a firstorder quasilinear equation with discontinuous flux, Arch. Rat. Mech. Anal., 195, 643-673, 2010.

[19] E. Yu. Panov, Ultra-parabolic equations with rough coeffcients. Entropy solutions and strong precompactness property, J. Math. Sci. (NY), 41, 159(2), 180-228, 2009.

[20] Y.J. Peng, Euler-Lagrange change of variables in conservation laws, Nonlin., 20, 1927-1953, 2007.

[21] D. Serre, Solutions à variations bornées pour certains systèmes hyperboliques de lois de conservation, J. Diff. Eqs., 68, 137-168, 1987.

[22] T. Tartar, Compensated compactness and applications to partial differential equations, in: Research Notes in Mathematics, Nonlinear Analysis and Mechanics, Heriot-Watt symposium, R.J. Knops (ed.), Pitman Press, London, 4, 1979.

[23] B. Temple, Global solution of the Cauchy problem for a class of $2 \times 2$ nonstrictly hyperbolic conservation laws, Adv. App. Math., 3, 335-375, 1982. 